\title{
Revista Colombiana de

\section{Capítulo 9. Ablación de extrasistolia ventricular y taquicardia ventricular en corazón sano}

\section{Chapter 9. Ablation of ventricular extrasystole and ventricular tachycardia in a healthy heart}

\author{
Jorge E. Marín a , Juan C. Díaz ${ }^{\mathrm{a}}$, Fermín García ${ }^{\mathrm{b}}$, Jorge E. Velásquez ${ }^{\mathrm{a}}$, \\ Julián M. Aristizábal ${ }^{a}$, Mauricio Duque ${ }^{a}$ y William Uribe ${ }^{a, *}$
}

\author{
a Servicio CES Cardiología, Medellín, Colombia \\ ${ }^{\mathrm{b}}$ Hospital of the University of Pennsylvania, Filadelfia, Estados Unidos
}

Recibido el 17 de noviembre de 2015; aceptado el 18 de enero de 2016

\section{Introducción}

La taquicardia ventricular idiopática (es decir, aquella que no se relaciona con isquemia o alteraciones estructurales cardiacas que generen áreas cicatriciales), representa aproximadamente el $10 \%$ de las taquicardias ventriculares. Dentro de este grupo de arritmias se incluyen las taquicardias ventriculares fascicular (dependientes de un mecanismo de reentrada; generalmente se originan cercanas al fascículo posterior), automática, paroxística inducida por el ejercicio y monomórfica repetitiva, esta última posiblemente la más común de todas ${ }^{1}$. Dentro de este último grupo se incluyen las taquicardias originadas en los tractos de salidas ventriculares, los músculos papilares, los anillos valvulares, las fasciculares y ocasionalmente otras taquicardias originadas en la cruz del corazón y en distintas partes del ventrículo derecho. A continuación, se definen los distintos tipos de taquicardia ventricular idiopática y se establecen conceptos que permiten su mapeo durante el estudio electrofisiológico.

\section{Taquicardias originadas en los tractos de salida ventricular}

En el caso de las taquicardias originadas en los tractos de salida ventricular (tanto en el ventrículo derecho como en el izquierdo), estas arritmias parecen estar relacionadas con mecanismos mediados por catecolaminas que involucran un aumento en el AMP y la liberación subsiguiente de calcio a partir del retículo sarcoplasmático. La liberación de calcio favorece la aparición de posdespolarizaciones tardías en presencia de catecolaminas, y son inhibidas por beta-bloqueadores, calcio-antagonistas, adenosina y maniobras vagales ${ }^{1-4}$.

\section{Extrasistolia y taquicardia de origen en el tracto de salida del ventrículo derecho}

La taquicardia ventricular originada en el tracto de salida del ventrículo derecho es la forma más común de taquicardia ventricular idiopática; representa aproximadamente el $70 \%$

*Autor para correspondencia.

Correo electrónico: william.uribe.md@gmail.com (W. Uribe). 
de los casos y es de mayor prevalencia en mujeres ${ }^{5-7}$. Típicamente se presenta entre la cuarta y quinta décadas de la vida y tiene como desencadenantes el estrés y el ejercicio. En general, los pacientes manifiestan palpitaciones como síntoma principal, sin embargo, también se han descrito dolor y opresión precordial, presíncope o síncope, disnea, fatiga, cansancio e intolerancia al ejercicio. Incluso hay casos reportados de tos asociada con extrasistolia ventricular, con resolución de los síntomas una vez se realiza ablación del foco arritmogénico ${ }^{8,9}$.

\section{Consideraciones anatómicas del tracto de salida del ventrículo derecho}

Se reconoce que la mayoría de las taquicardias y complejos ventriculares prematuros (extrasístoles) de etiología no isquémica se localizan en el tracto de salida del ventrículo derecho, de ahí que el enfoque inicial se base en la determinación de las características morfológicas de las arritmias originadas en esta zona. Desde el punto de vista anatómico, el tracto en mención tiene una posición anterior, izquierda, y ligeramente superior respecto al tracto de salida del ventrículo izquierdo y está delimitado en su parte superior por la válvula pulmonar mientras que su parte inferior por la parte alta del anillo tricúspide (correspondiente a la zona del haz de His). Su pared medial atañe en su porción más baja al septum interventricular, en tanto que la superior y anterior no corresponde a ninguna estructura en el ventrículo izquierdo (es decir, una punción o ruptura en este punto comunicará directamente hacia el espacio pericárdico). Es importante tener esto en mente al momento de manipular los catéteres en esta zona, especialmente si se tiene en cuenta que la mayoría de las arritmias del tracto de salida del ventrículo derecho se originan en esta localización ${ }^{10,11}$. La porción posterior alta está en continuidad directa con la zona de la cúspide aórtica derecha, hecho que explica las similitudes electrocardiográficas entre las extrasístoles originadas en el tracto de salida del ventrículo derecho y las cúspides (ver adelante). Su pared lateral está dada por la pared libre del ventrículo derecho, una estructura más delgada y, por tanto, más expuesta a lesiones mecánicas que pueden llevar a complicaciones durante los procedimientos de mapeo y ablación (fig. 1).

Desde un enfoque tridimensional el tracto de salida del ventrículo derecho se divide en: anterior y posterior, derecho e izquierdo, y superior e inferior. La mitad anterior de este tracto evaluada mediante fluoroscopia en proyección oblicua anterior izquierda a 60 grados, define la posición como la cara anterior (pared libre), en tanto que la mitad posterior como la cara posterior (pared septal). Cuando se valora en la proyección oblicua anterior derecha a 30 grados, la mitad posterior del tracto de salida del ventrículo derecho se define como posterolateral, mientras que la mitad anterior como anterolateral. El área a $1 \mathrm{~cm}$ de la válvula pulmonar se define como la cara superior (la cara distal apenas debajo de la válvula pulmonar), y el área a más de $1 \mathrm{~cm}$ se define como la cara inferior. Esto significa que el tracto de salida del ventrículo derecho consta de ocho subdivisiones. Además de lo expuesto previamente, es vital el análisis en conjunto con las demás estructuras anatómicas que pueden igualmente ser origen de taquicardio-ventricular no isquémico y para las que el elec-

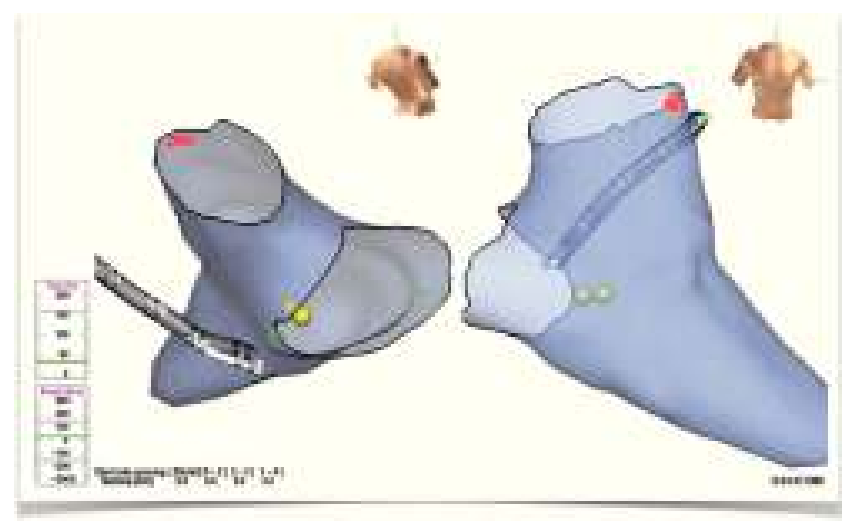

Figura 1 Reconstrucción anatómica tridimensional utilizando el sistema EnSite basado en una angiotomografía con fusión a través del software específico del sistema 3-D de St. Jude ${ }^{\circledR}$. Los puntos amarillos muestran el haz de His y los rojos el sitio exitoso de ablación. El catéter en blanco (real) y gris (sombra) se encuentra en el seno coronario.

trocardiograma de superficie no es completamente específico cuando se trata de localizar el foco de la arritmia ${ }^{12}$.

\section{Características electrocardiográficas}

Dada la posición anatómica del trato salida del ventrículo derecho dentro del tórax, las extrasístoles originadas en esta región tienen un eje inferior (es decir, positivas en DII, DIII y aVF) con una imagen de bloqueo de rama izquierda del haz de His (BRIHH), cuya transición en el plano precordial sucede por lo general después de $\mathrm{V} 3^{13}$. Debido a su cercanía con el sistema de conducción, las arritmias originadas de la pared septal del tracto de salida del ventrículo derecho tendrán, por lo general, complejos angostos en comparación con aquellas que se producen en la pared lateral, las cuales incluso pueden llegar a tener complejos mellados siendo claramente positivas tanto en DI como en aVL. Por otro lado, la porción más anterior del tracto de salida del ventrículo derecho está ubicada hacia la izquierda del tórax, por lo cual las extrasístoles originadas en esta región tienden a ser negativas en las derivaciones DI y aVL, mientras que las que se originan hacia la porción posterior del mismo tracto son ligeramente positivas en $\mathrm{V} 1$, manteniendo su negatividad (o en ocasiones en forma de W, principalmente a medida que se desciende en el tracto) en aVL. Por su parte, a pesar de que se han descrito fibras musculares que se extienden desde el tracto de salida del ventrículo derecho a través de la válvula pulmonar, las arritmias ventriculares originadas en el plano supravalvular son infrecuentes; no obstante, cuando se realiza ablación en un punto infravalvular y se modifica la morfología del QRS haciéndose más inferior, se debe explorar el plano supravalvular para descartar un origen en esta zona.

Es así como mediante el análisis del electrocardiograma de superficie es factible inferir el posible origen de una extrasístole y de esta manera ayudar durante la tarea de mapeo y ablación con catéter. No obstante, se debe tener en cuenta que las variaciones en la posición anatómica del corazón en el tórax y la forma en la cual se ubiquen las derivaciones en 
el plano precordial y frontal durante la toma del electrocardiograma, hacen que estos patrones no sean $100 \%$ confiables al momento de determinar el origen de la arritmia.

\section{Utilidad del mapeo tridimensional}

Se parte del hecho de que el corazón es una estructura tridimensional y el análisis anatómico que permite la fluoroscopia en dos planos tiene limitaciones claras, lo cual impacta directamente en el resultado clínico. La cercanía de las estructuras de los tractos de salida (tracto de salida del ventrículo derecho e izquierdo, senos de Valsalva y cima del ventrículo izquierdo) obliga a tener claridad respecto a la relación anatómica de estas estructuras y realizar un mapeo juicioso y detallado de las distintas áreas con el fin de determinar el origen de la arritmia ${ }^{12}$. Tradicionalmente, las técnicas de mapeo convencional incluyen un mapeo de activación (idealmente con una precocidad electrográfica a QRS mayor a $30 \mathrm{~ms}$ ) y de topoestimulación (se busca lograr una concordancia entre el QRS estimulado y el QRS de la arritmia en las 12 derivaciones del electrocardiograma) ${ }^{13}$. Si bien estas técnicas proporcionan ayuda valiosa, tienen limitaciones importantes por lo cual su uso como herramienta única para la determinación del origen de la arritmia. En el caso de la topoestimulación se ha descrito que aun diferencias mínimas en los QRS pueden representar distancias de más de $5 \mathrm{~mm}$ entre el sitio de estimulación y el origen real de la arritmia ${ }^{14}$. Es aquí donde los sistemas de mapeo tridimensional desempeñan un papel importante ya que permiten ubicar con gran precisión la posición del catéter dentro de la cavidad ventricular, lo cual facilita la navegación al interior de los tractos de salida y la marcación de los sitios que han sido evaluados.

El punto de partida para el mapeo tridimensional es la reconstrucción virtual de la anatomía del tracto de salida del ventrículo derecho, teniendo en cuenta que las estructuras anatómicas adyacentes (tracto de salida del ventrículo izquierdo, senos de Valsalva, raíz aórtica, arteria pulmonar, cima del ventrículo izquierdo) deben tenerse en cuenta cuando la precocidad del electrograma local respecto al inicio del QRS, no es suficientemente buena (es decir, $>30 \mathrm{~ms}$ ). Este punto es fundamental ya que un error común es asumir que el punto de mayor precocidad es el origen de la arritmia. En realidad, este representa el punto más precoz en la cámara estudiada, mas no necesariamente el origen de la arritmia. Por ejemplo, al mapear desde el tracto de salida del ventrículo derecho una extrasístole ventricular que se origine en la cúspide coronaria derecha, se observará como sitio de activación inicial el lugar por donde el estímulo pasa de la cúspide derecha hacia el ventrículo derecho. Claramente, este es el sitio más precoz en el ventrículo derecho, sin que sea el verdadero origen de la arritmia. La ablación entonces será fallida, y no se logrará la desaparición de la extrasístole ventricular a pesar de que por calentamiento se obtenga supresión transitoria. Es en estos casos donde se aprecia la capacidad del mapeo electroanatómico para discriminar entre dos zonas que se encuentran separadas por menos de $1 \mathrm{~cm}$.

Las técnicas de mapeo tridimensional incluyen técnicas de contacto como el EnSite NavX (St. Jude Medical, St Paul, Minnesota, Estados Unidos) y el sistema CARTO (Biosense Webster, Diamond Bar, California, Estados Unidos), que han de- mostrado su utilidad para la ablación en este tipo de arritmias (fig. 2) ${ }^{15,16}$. También existen sistemas de mapeo de no contacto (EnSite 3000 Non contact mapping system, Endocardial Solutions, St Paul, Minnesota, Estados Unidos) que igualmente han demostrado efectividad clínica ${ }^{17,18}$. Este tipo de mapeo está indicado para taquicardias difíciles de reinducir o cuando las extrasístoles ventriculares son escasas. En la actualidad, está aprobado su uso en taquiarritmias auriculares y ventriculares de las cavidades derechas.

\section{Extrasistolia y taquicardia de origen en cúspides coronarias (senos de Valsalva)}

En comparación con el tracto de salida del ventrículo derecho, el del ventrículo izquierdo es un sitio que da origen a tan solo el 10 al $15 \%$ de las arritmias sin diferencias significativas de acuerdo con el género del paciente ${ }^{5,19}$. La mayoría de estas proviene de la región de las cúspides aórticas, y es más común el origen en la cúspide izquierda ${ }^{19,20}$.

\section{Consideraciones anatómicas del tracto de salida del ventrículo izquierdo}

Desde el punto de vista anatómico, el botón aórtico tiene una ubicación central con el tracto de salida del ventrículo derecho ubicado por delante, discretamente superior y a la derecha. Está compuesto por tres cúspides: la derecha, donde normalmente se origina la coronaria homónima; la izquierda, de ubicación ligeramente superior y anterior y que da origen al tronco principal izquierdo, y la no coronaria, de ubicación posterior (está en relación con la aurícula izquierda), siendo esta la más inferior de todas. A pesar de que se han descrito arritmias ventriculares en las tres cúspides ${ }^{21,22}$, en nuestra experiencia no se tienen documentadas arritmias ventriculares originadas en la no coronaria; son las cúspides derechas e izquierdas las que tienen estrecha relación con el miocardio del ventrículo izquierdo, y por tanto es aquí donde se origi-

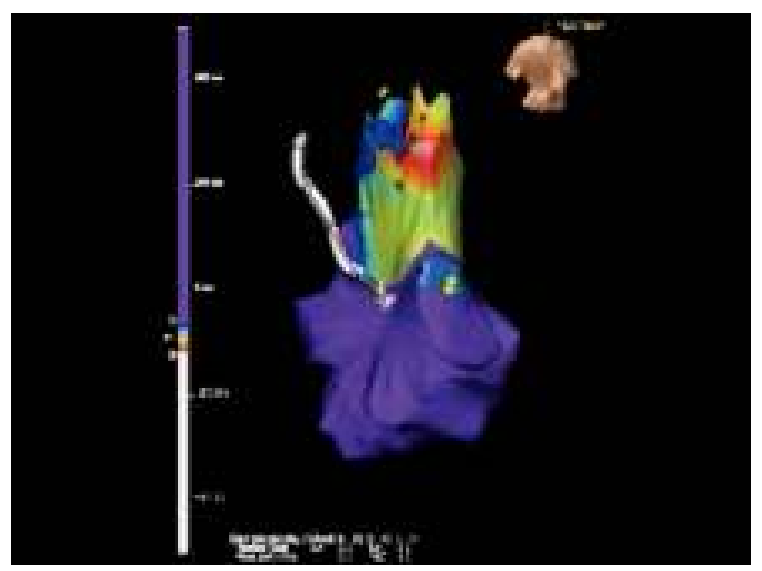

Figura 2 Mapa de activación del tracto de salida del ventrículo derecho para el que se usó el sistema EnSite. Se observa la precocidad de una señal representada en el esquema de colores, en donde el blanco es el sitio de mayor precocidad (en este caso, a nivel de la pared posterior y lateral del tracto de salida) y el morado corresponde a sitios más tardíos. De esta forma, se identifica visualmente el objetivo facilitando así la ablación. 
nan la mayoría de las arritmias. En la unión entre la cúspide coronaria derecha y la no coronaria, discurre el haz de His, relación que debe tener presente al momento de efectuar ablaciones en esta región con el objetivo de evitar daños al sistema de conducción (esta relación es tan cercana, que se ha descrito la ablación de vías accesorias parahisianas desde esta zona) ${ }^{23}$.

\section{Características electrocardiográficas}

Debido a su cercanía (fig. 3), las arritmias que se generan en las cúspides comparten varias características electrocardiográficas con las que se originan en la porción septal del tracto de salida del ventrículo derecho (fig. 4):

- Eje inferior.

- Morfología del BRIHH.

- QRS relativamente estrecho.

No obstante, dada su ubicación en el tracto de salida del ventrículo izquierdo, la transición en el plano precordial se produce por lo general antes de V3 (para la cúspide izquierda, entre V1 y V2, mientras que para la cúspide derecha por lo general es entre V2 y V3) ${ }^{24}$. Cuando la transición ocurre en V3, la comparación de la transición de la arritmia con relación a la del ritmo sinusal, es útil para definir un origen en el tracto de salida del ventrículo derecho vs. el izquierdo ${ }^{13}$. Es así como las que se originan en este último tienen una transición más temprana que la del ritmo sinusal, en tanto que una transición tardía favorece un origen en el tracto de salida del ventrículo derecho.

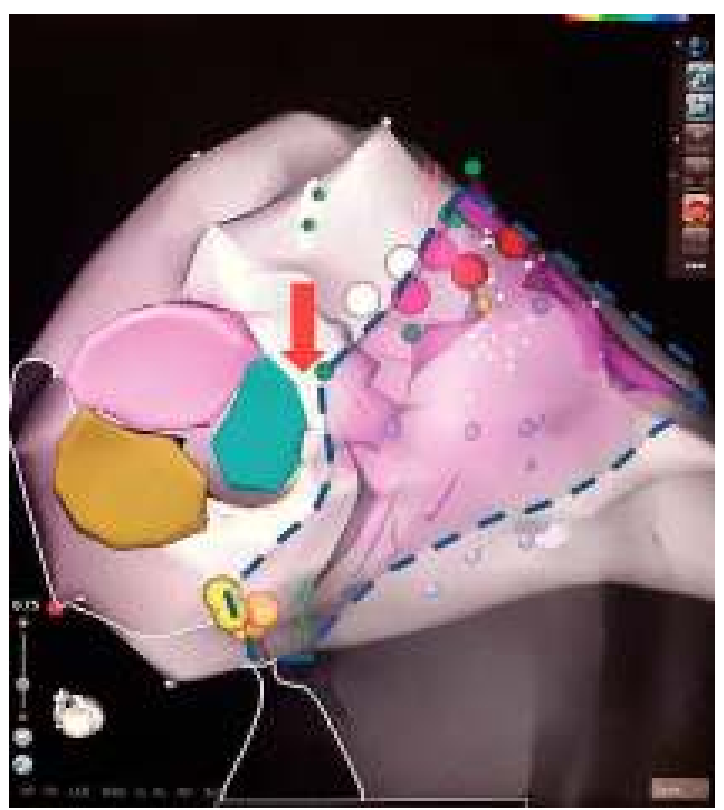

Figura 3 Relación entre el tracto de salida izquierdo y el derecho (zona delimitada por la línea punteada). Obsérvese la cercanía de la cúspide coronaria derecha (azul claro) y el tracto de salida del ventrículo derecho, los cuales están separados por unos pocos milímetros (flecha roja). Esto explica las similitudes existentes entre las extrasístoles originadas en los tractos de salida del ventrículo derecho e izquierdo.

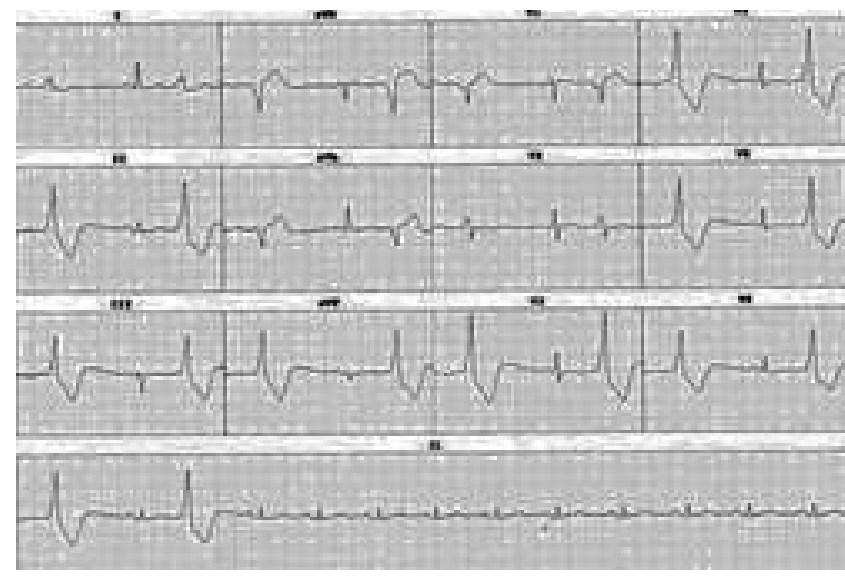

Figura 4 Extrasístole ventricular originada en la cúspide coronaria derecha. Comparte varias características con el tracto de salida del ventrículo derecho (explicado por su cercanía anatómica) como QRS relativamente estrecho (en comparación con las extrasístoles que se originan en la pared libre de los ventrículos), eje inferior e imagen de bloqueo de rama izquierda en V1. No obstante, la transición se produce entre V2-V3 y ocurre antes que la transición del ritmo sinusal, lo cual apunta hacia un origen en cúspide. Obviar esta relación puede llevar a errores de interpretación en los mapas de activación, ya que a nivel del tracto de salida del ventrículo derecho se observará una zona precoz (sitio de entrada de la extrasístole al ventrículo derecho) pero las aplicaciones de radiofrecuencia en esta zona serán inefectivas.

\section{Utilidad del mapeo tridimensional}

Al utilizar cualquiera de los sistemas de mapeo previamente descritos, el acceso recomendado es la arteria femoral ya que de esta manera se logra avanzar del catéter de ablación hasta la raíz aórtica. En este sitio, el uso de ecocardiografía intracardiaca y reconstrucción tridimensional con mapeo no fluoroscópico permite definir con claridad las diferentes estructuras anatómicas, particularmente los senos de Valsalva y los ostium coronarios, de modo que se puede establecer su relación con la región de interés para proporcionar una intervención segura y efectiva para el paciente ${ }^{25}$.

Con ayuda del sistema de mapeo tridimensional se procede a realizar un mapa anatómico tanto de la válvula aórtica como de los senos de Valsalva, los ostium coronarios derecho e izquierdo, el tracto de salida del ventrículo izquierdo y el segmento proximal de la aorta ascendente. A partir de este mapa se procede a la adquisición de puntos de activación que permiten, de acuerdo con la precocidad, realizar un mapa de activación y así localizar el foco de la taquicardia (fig. 5$)^{26}$.

Los hallazgos del mapeo de activación se deben corroborar con la topoestimulación a fin de optimizar el blanco de ablación y el resultado clínico (fig. 6). Debido a la pequeña cantidad de miocardio presente en esta zona, por lo general la estimulación requiere un aumento del voltaje de salida con el objetivo de asegurar la captura. Esto a su vez genera dificultades significativas, ya que al emplear estos estímulos se puede capturar el miocardio lejano, lo cual arrojaría una topoestimulación muy distinta a pesar de estar sobre el origen de la arritmia. 


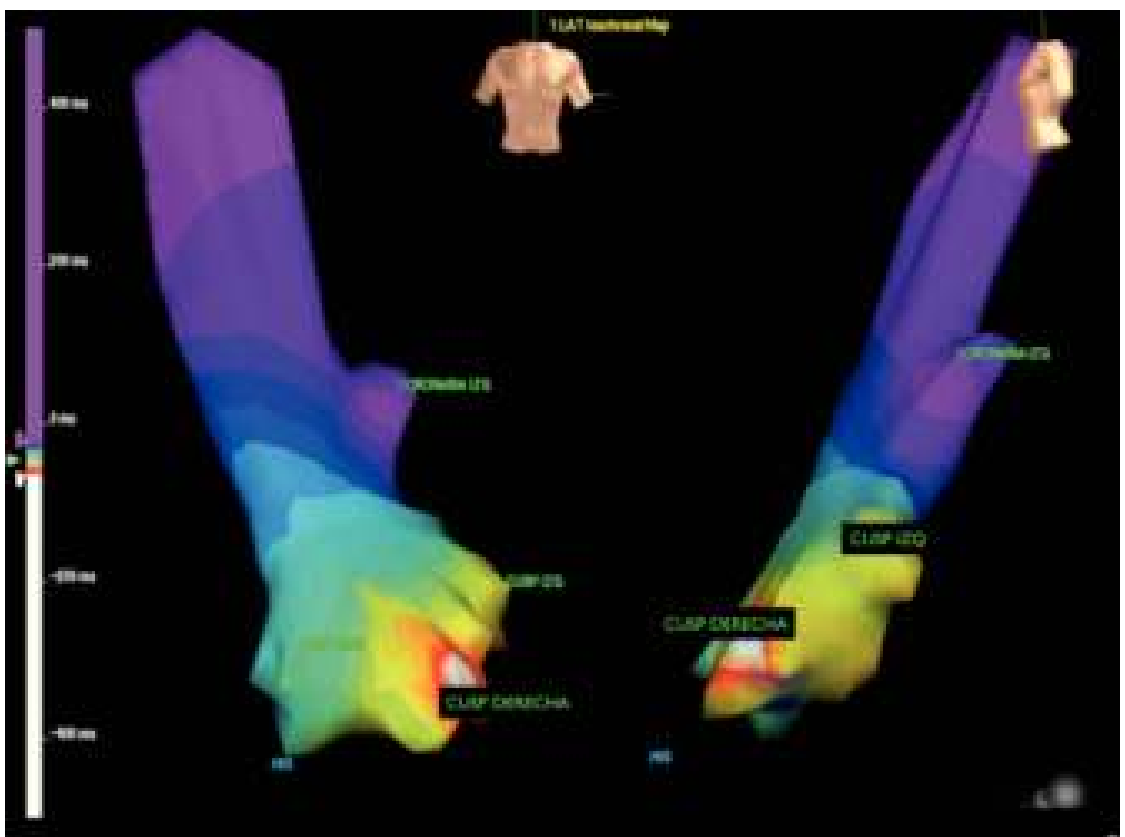

Figura 5 Anatomía del botón aórtico establecida mediante reconstrucción tridimensional con sistema EnSite. Se observa cómo la cúspide derecha es más anterior mientras que la izquierda tiene una ubicación más superior, lo que permite su identificación en fluoroscopia. La no coronaria (no representada en este gráfico) es la más posterior e inferior. En esta imagen se observa además un mapa de activación que permite ubicar el origen de la taquicardia a nivel de la cúspide derecha.

\section{Extrasistolia y taquicardia de origen en la continuidad mitro-aórtica}

La continuidad mitro-aórtica como sitio de origen de taquicardias ventriculares idiopáticas, es infrecuente. El enfoque

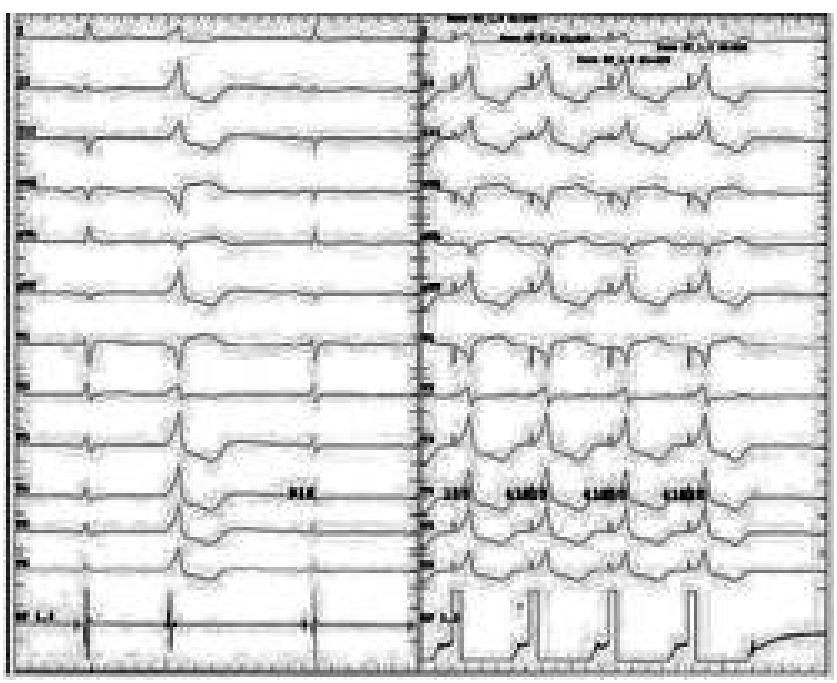

Figura 6 Topoestimulación a nivel de la cúspide izquierda con reproducción de la morfología de la extrasístole en las 12 derivaciones del electrocardiograma. Durante la topoestimulación es importante estimular a una longitud de ciclo similar al intervalo de acoplamiento de la extrasístole con el fin de evitar cambios en el patrón de activación ventricular que puedan generar variaciones en la morfología, ya que lograr una morfología exactamente igual es fundamental durante esta maniobra. en estos casos es similar al descrito previamente para las cúspides coronarias. Se inicia con el análisis del electrocardiograma, en búsqueda de características electrocardiográficas que apuntan a esta zona:

- Presencia de complejos qR en V1.

- Complejos RS o rs en DI.

El acceso vascular se hace a nivel de la arteria femoral común derecha, sitio por el cual se avanza el catéter de mapeo hasta la raíz aórtica y hasta la cavidad ventricular a fin de posicionarlo en la continuidad. La falta de estabilidad del catéter y las dificultades en la maniobrabilidad del mismo en esta zona, hacen que la ablación de estas arritmias sea técnicamente más difícil que la de otras zonas. Todas las extrasístoles de cualquier origen pueden producir taquicardiomiopatía y recientemente se la ha relacionado con origen en esta zona (figs. 7 y 8$)^{26}$.

\section{Beneficios de los sistemas de mapeo tridimensional en la ablación de arritmias ventriculares idiopáticas}

Como ya se anotó, el análisis del electrocardiograma orienta hacia el posible origen de una arritmia ventricular; no obstante, está supeditado a una gran fuente de variaciones entre las que se encuentran la posición del corazón dentro de tórax y la ubicación de los electrodos en la superficie del paciente. Por otro lado, teniendo en cuenta que los tractos de salida están compuestos por un sinnúmero de estructuras con una gran superposición, la evaluación fluroscópica como única herramienta para la ubicación del origen de las arritmias es insuficiente para llevar a cabo un procedimiento cu- 


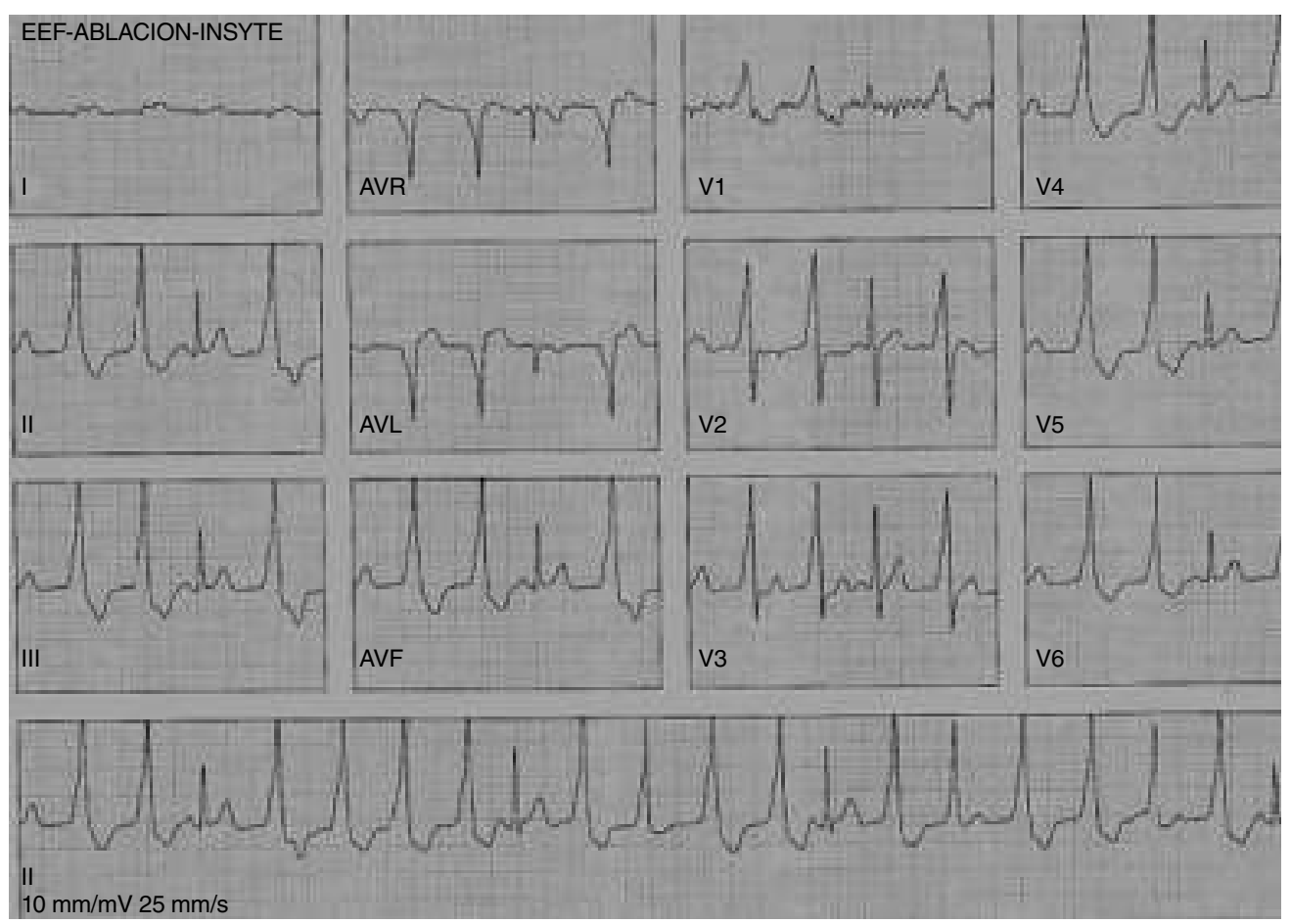

Figura 7 Taquicardia ventricular originada en la continuidad mitro-aórtica.

rativo. Es necesario el uso de otras tecnologías que permitan diferenciar entre distintas estructuras y establecer la relación entre ellas, y es aquí donde los sistemas de mapeo tridimensional desempeñan un papel preponderante en la ablación de arritmias ventriculares idiopáticas.

Con relación al mapeo tridimensional, los sistemas disponibles en Colombia, CARTO y NavX, están claramente indicados para el mapeo de taquicardia ventricular no isquémica con localización en los tractos de salida del ventrículo derecho e izquierdo, las cúspides coronarias y el epicardio.

De otra parte, existen varias ventajas relacionadas con su uso:

1. Reducción de la exposición a radiación al paciente, así como al personal médico y paramédico.
2. Mayor precisión anatómica que la que permite el mapeo fluoroscópico (con una resolución espacial de menos de 1 $\mathrm{mm}$ para los dos sistemas disponibles).

3. Reducción de la duración de los procedimientos.

4. Representación de la ubicación de estructuras adyacentes importantes, como por ejemplo los ostium coronarios.

5. Registro visual de los sitios donde se realizó ablación, evitando repetir lesiones cuando son ineficaces o repitiéndolas cuando se logró el objetivo terapéutico.

No existen desventajas asociadas al uso de sistemas tridimensionales siempre y cuando el operador comprenda el funcionamiento del sistema. Cuando no se tienen en cuenta preceptos básicos de electrofisiología, la presencia de una zona

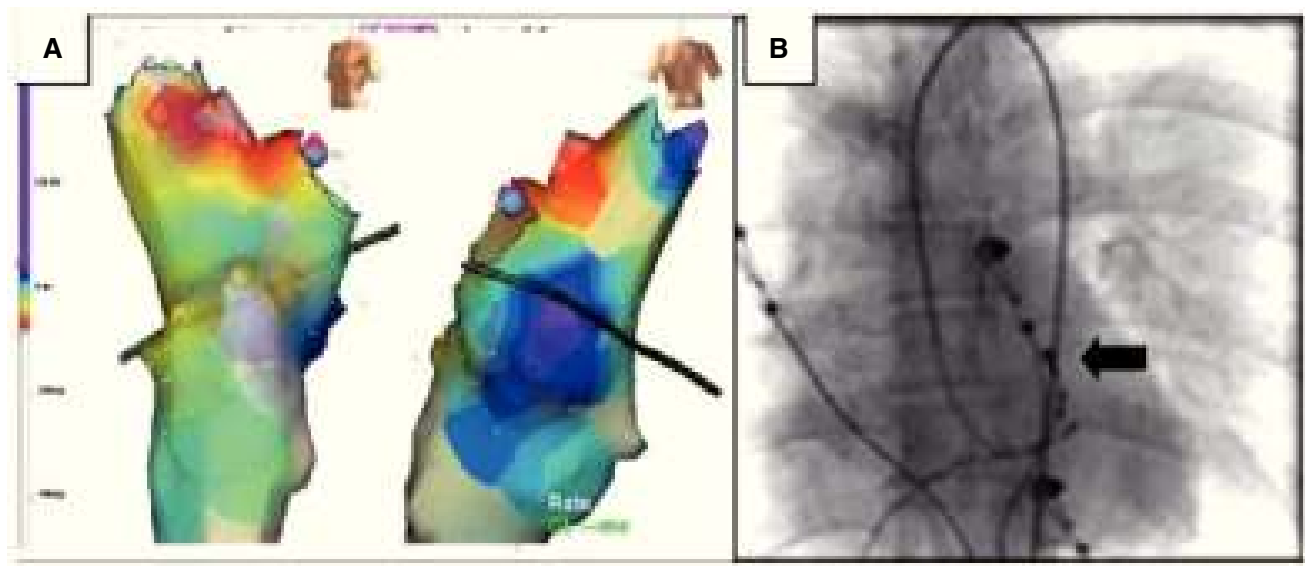

Figura 8 A. Reconstrucción electroanatómica del ventrículo izquierdo con mapa de activación de la taquicardia ventricular, con ablación exitosa en la continuidad mitro-aórtica. B. Localización del catéter de ablación en proyección antero-posterior en la fluoroscopia. 
temprana en una cavidad puede ser interpretada como el origen de la misma, olvidando lo que se había establecido sobre el origen en cavidades y estructuras adyacentes y los sitios por donde el impulso pasa a otras cavidades.

\section{Conclusiones}

Las arritmias ventriculares idiopáticas (entendiendo de esta manera las arritmias que se producen en ausencia de una alteración cardiaca estructural) se originan por lo general a nivel de los tractos de salida, siendo más común el origen en el tracto de salida del ventrículo derecho. Debido a la proximidad anatómica de dichas estructuras, las características electrocardiográficas tienen un grado de superposición significativa y se requieren criterios electrocardiográficos específicos con el fin de tratar de dilucidar su origen. No obstante, dadas las variaciones que pueden acaecer durante la toma del electrocardiograma (dadas por las diferencias en la posición del corazón dentro del tórax o de la ubicación de los electrodos sobre el tórax del paciente), estos criterios solo brindan una idea acerca del origen mas no de la ubicación exacta del mismo. Con base en las limitaciones de la fluoroscopia para establecer la posición de estructuras compuestas por tejidos blandos (como es el caso de las cámaras cardiacas) los sistemas de navegación tridimensional ofrecen una oportunidad única para el mapeo de las arritmias ventriculares, pues disminuyen de manera significativa los tiempos de fluoroscopia y de procedimiento y al mismo tiempo mantienen una gran capacidad de discriminación espacial, con una resolución de $<1 \mathrm{~mm}$. Adicionalmente, permiten establecer la relación de la zona de ablación con estructuras adyacentes, con lo cual se reduce el riesgo de complicaciones por ablación inadvertida en lugares indeseados (por ejemplo, un ostium coronario). Por esta razón los sistemas de mapeo no fluoroscópico han ganado un lugar importante en el tratamiento curativo de las arritmias ventriculares.

\section{Recomendaciones}

\section{Clase I}

- El mapeo tridimensional se indica en la ablación de arritmias ventriculares idiopáticas en paciente en quienes se desea disminuir la exposición a los rayos $X$.

- El mapeo tridimensional se indica en la ablación de arritmias ventriculares idiopáticas en pacientes con recurrencias luego de ablación por sistemas convencionales.

\section{Clase IIA}

- El mapeo tridimensional puede recomendarse en la ablación de arritmias ventriculares idiopáticas como estrategia inicial.

\section{Bibliografía}

1. Lerman BB, Stein K, Engelstein ED, et al. Mechanism of repetitive monomorphic ventricular tachycardia. Circulation. 1995; 92:421-9.
2. Lerman BB. Response of nonreentrant catecholamine-mediated ventricular tachycardia to endogenous adenosine and acetylcholine. Evidence for myocardial receptor-mediated effects. Circulation. 1993;87:382-90.

3. Lerman BB, Belardinelli L, West GA, Berne RM, DiMarco JP. Adenosine-sensitive ventricular tachycardia: evidence suggesting cyclic AMP-mediated triggered activity. Circulation. 1986; 74:270-80.

4. Lerman BB, Stein KM, Markowitz SM. Mechanisms of idiopathic left ventricular tachycardia. J Cardiovasc electrophysiol. 1997; 8:571-83.

5. Nakagawa M, Takahashi N, Nobe S, et al. Gender differences in various types of idiopathic ventricular tachycardia. J Cardiovasc Electrophysiol. 2002;13:633-8.

6. Badhwar N, Scheinman MM. Idiopathic ventricular tachycardia: Diagnosis and management. Curr Prob Cardiol. 2007;32:7-43.

7. Pellegrini $\mathrm{CN}$, Scheinman MM. Clinical management of ventricular tachycardia. Curr Prob Cardiol. 2010;35:453-504.

8. Hasdemir C, Musayev O, Kehribar DY, Kartal Y, Can LH. Chronic cough and tachycardia-induced cardiomyopathy in a patient with idiopathic frequent, monomorphic premature ventricular contractions. PACE. 2013;36:e156-8.

9. Stec SM, Grabczak EM, Bielicki P, et al. Diagnosis and management of premature ventricular complexes-associated chronic cough. Chest 2009;135:1535-41.

10. Latif S, Dixit S, Callans DJ. Ventricular arrhythmias in normal hearts. Cardiol Clin. 2008;26:367-80, vi.

11. Movsowitz C, Schwartzman D, Callans DJ, et al. Idiopathic right ventricular outflow tract tachycardia: narrowing the anatomic location for successful ablation. Am Heart J. 1996;131: 930-6.

12. Dixit S, Lin D, Marchlinski FE. Ablation of ventricular outflow tract tachycardias. In: Huang SK, Wood MA, editors. Catheter Ablation of Cardiac Arrhythmias. 2 ed. Philadelphia, PA: Saunders; 2011. p. 446-62.

13. Garcia F, Uribe W. Correlación anatómica-electrocardiográfica de las arritmias originadas en los tractos de salida ventriculares. In: Uribe W, editor Electrocardiografía Clínica: de lo básico a lo complejo. 1. ${ }^{a}$. ed. Bogotá: Editorial Distribuna; 2014. p. 597-610.

14. Azegami K, Wilber DJ, Arruda M, Lin AC, Denman RA. Spatial resolution of pacemapping and activation mapping in patients with idiopathic right ventricular outflow tract tachycardia. J Cardiovasc Electrophysiol. 2005;16:823-9.

15. Lerman BB, Stein KM, Markowitz SM, Mittal S, Slotwiner DJ. Ventricular arrhythmias in normal hearts. Cardiology Clin. 2000;18:265-91.

16. Miles WM. Idiopathic ventricular outflow tract tachycardia: where does it originate? J Cardiovasc Electrophysiol. 2001;12: 536-7.

17. Friedman PA, Asirvatham SJ, Grice S, et al. Noncontact mapping to guide ablation of right ventricular outflow tract tachycardia. J Am Coll Cardiol. 2002;39:1808-12.

18. Friedman PA, Beinborn DA, Schultz J, Hammill SC. Ablation of noninducible idiopathic left ventricular tachycardia using a noncontact map acquired from a premature complex with tachycardia morphology. PACE. 2000;23:1311-4.

19. Yamada T, McElderry HT, Doppalapudi H, et al. Idiopathic ventricular arrhythmias originating from the aortic root prevalence, electrocardiographic and electrophysiologic characteristics, and results of radiofrequency catheter ablation. J Am Coll Cardiol. 2008;52:139-47.

20. Yamada T, Yoshida N, Murakami Y, et al. Electrocardiographic characteristics of ventricular arrhythmias originating from the junction of the left and right coronary sinuses of Valsalva in the aorta: the activation pattern as a rationale for the electrocardiographic characteristics. Heart Rhythm. 2008;5: 184-92. 
21. Hlivak P, Peichl P, Cihak R, Wichterle D, Kautzner J. Catheter ablation of idiopathic ventricular tachycardia originating from myocardial extensions into a noncoronary aortic cusp. J Cardiovasc Electrophysiol. 2012;23:98-101.

22. Sayah S, Shahrzad S, Moradi M, Haghjoo M. Electrocardiographic, electrophysiologic, and anatomical features of ventricular tachycardia originating from noncoronary cusp. J Electrocardiol. 2012;45:170-5.

23. Xu G, Liu T, Liu E, Ye L, Shehata M, Wang X, et al. Radiofrequency catheter ablation at the non-coronary cusp for the treatment of para-hisian accessory pathways. Europace. 2015;17:962-8.
24. Uribe W, Astudillo V, Medina L, et al. Taquicardia ventricular proveniente de las cúspides coronarias, características electrocardiográficas, electrofisiológicas y resultados de ablación por radiofrecuencia, validación de un algoritmo diagnóstico. Revista Iberoamericana de Arritmología. 2010:134-46.

25. Hachiya H, Aonuma K, Yamauchi Y, Igawa M, Nogami A, lesaka Y. How to diagnose, locate, and ablate coronary cusp ventricular tachycardia. J Cardiovasc Electrophysiol. 2002;13:551-6.

26. Mora G, Romero N, Rendón I. Tachycardiomyopathy: a rare manifestation of left ventricular outflow tract tachycardia. Treatment with radiofrequency catheter ablation. Indian Pac Electrophysiol J. 2013;13:38-42. 